\title{
Search for hydrogen-helium molecular species in space
}

\author{
E. Zicler ${ }^{1}$, O. Parisel ${ }^{1}$, F. Pauzat ${ }^{1}$, Y. Ellinger ${ }^{1}$, M.-C. Bacchus-Montabonel ${ }^{2}$, and J.-P. Maillard ${ }^{3}$ \\ 1 Sorbonne Universités, UPMC Univ. Paris 06, UMR - CNRS 7616, Laboratoire de Chimie Théorique, 75005 Paris, France \\ e-mail: yves. ellinger@upmc.fr \\ 2 Univ. Lyon, Université Claude Bernard Lyon 1, CNRS, Institut Lumière Matière, 69622 Villeurbanne, France \\ e-mail: marie-christine.bacchus@univ-lyon1.fr \\ 3 Sorbonne Universités, UPMC Univ. Paris 06 et CNRS, UMR 7095, Institut d'Astrophysique de Paris, 75014 Paris, France \\ e-mail: jean-pierre.maillard@iap.fr
}

Received 25 June 2017 / Accepted 27 July 2017

\begin{abstract}
Context. Helium, the second most abundant element in the Universe, with a relative abundance of $\mathrm{He} / \mathrm{H} \sim 1 / 10$, has never been observed in any other form than that of a neutral atom $(\mathrm{He})$ or an ion $\left(\mathrm{He}^{+}\right)$in the interstellar medium. Since He is a noble gas its non-observation as part of neutral molecular systems is understandable, but it is very surprising for a positively charged species such as $\mathrm{HeH}^{+}$that is a stable diatomic ion whose spectral signatures are well known in the laboratory.

Aims. This non-observation, even in hydrogen rich regions, could imply that $\mathrm{HeH}^{+}$is not a proper target and that alternatives have to be considered, such as small $\mathrm{HeH}_{n}^{+}$clusters. The present study aims at finding whether the leading term $\mathrm{HeH}_{3}^{+}$fulfills the conditions required.

Methods. We addressed the question with state-of-the-art numerical simulations. We determined a two-dimension ab initio potential energy surface (PES) of the $\mathrm{HeH}_{3}^{+}$cluster along the $\mathrm{He}_{2} . . \mathrm{H}_{3}^{+}$and $\mathrm{HeH}^{+} \ldots \mathrm{H}_{2}$ reaction coordinates. The calculations rely on complete active space configuration interaction followed by a second order perturbation treatment (CAS-PT2). This surface was used for the evaluation of the two radiative associations rate constants by means of a quantum treatment of the collision between the interacting fragments.

Results. These calculations show unambiguously that $\mathrm{HeH}_{3}^{+}$is the most stable point on the corresponding global PES. Then, we determined the rate constants of the radiative associations $\mathrm{HeH}^{+}+\mathrm{H}_{2}$ and $\mathrm{He}+\mathrm{H}_{3}^{+}$leading to $\mathrm{HeH}_{3}^{+}$.

Conclusions. Significative values were obtained that reach up to $2 \times 10^{-18} \mathrm{~cm}^{3} \mathrm{~s}^{-1}$, which should stimulate new tentatives to detect molecular helium in astrophysical objects.
\end{abstract}

Key words. ISM: molecules - ISM: abundances

\section{Introduction}

$\mathrm{HeH}^{+}$is composed of the two most abundant elements of the Universe and is the simplest heteronuclear ion that can exist. It is also believed to be the first molecular compound that appears in the Universe (Lepp et al. 2002). Moreover, the stars formed from the primordial material, when no heavy element exists (Hirasawa 1969; Dalgarno \& Lepp 1987), should contain $\mathrm{HeH}^{+}$, which would obviously play a role in their formation and, later, in their evolution. But despite all the attention focused on this element, $\mathrm{HeH}^{+}$has never been detected unambiguously after 30 years of fruitless attempts, although its spectral signatures are well known in the laboratory since Hogness \& Lunn (1925).

Dabrowski \& Herzberg (1978) were the first to conjure up the idea of a possible detection of $\mathrm{HeH}^{+}$in astrophysical environments. From the calculation of the vibrational and rotational energy levels of the ground electronic state of $\mathrm{HeH}^{+}$, these authors deduced that it could have been formed by radiative association. At about the same time Black (1978) and Flower \& Roueff (1979) calculated the abundance of $\mathrm{HeH}^{+}$together with its emission line intensities in gaseous nebulae, which highlighted the difficulty of its detection.

In 1982, Roberge \& Dalgarno (1982) listed the formation and destruction mechanisms of $\mathrm{HeH}^{+}$in physical plasmas. Those calculations showed that, in principle, $\mathrm{HeH}^{+}$could be detected, in planetary nebulae (PN) and in dense molecular clouds subject to X-ray and extreme UV ionization. Later, Harris et al. (2004) thought it was possible to find $\mathrm{HeH}^{+}$in the atmospheres of helium-rich white dwarfs and stated that it should be the dominant positive ion among such stars, and this, over a wide range of temperatures and densities. Then, Engel et al. (2005), by calculating the spectra of $\mathrm{HeH}^{+}$and opacity data, proposed that $\mathrm{HeH}^{+}$ could be detected in cold helium stars. Recent chemical models, such as that developed by Bovino et al. $(2011,2012)$ have also predicted that $\mathrm{HeH}^{+}$could be the most abundant molecule of the post-recombination era and could survive so as to be observed at low redshifts.

There has been several attempts at detecting $\mathrm{HeH}^{+}$in the interstellar medium, but in vain, contrary to $\mathrm{ArH}^{+}$recently observed (Barlow et al. 2013). The first attempt was carried out by Moorhead et al. (1988) who tried to detect $\mathrm{HeH}^{+}$in $\mathrm{PN}$ NGC 7027; this PN is known for having one of the hottest central stars (Gruenwald \& Péquignot 1989; Middlemass 1990) surrounded by an envelope of ionized gas that is rich in atomic hydrogen and helium. Theoretically, this situation would ensure a sufficient formation rate of the ion. However, the detection attempts were never conclusive. Miller et al. (1992) searched again by looking at the spectra of supernova 1987A. But, to the best of our knowledge, the observation has never been confirmed and neither has that by Liu et al. (1997) carried out again towards PN 
NGC 7027. Lately, Zinchenko et al. (2011) searched for $\mathrm{HeH}^{+}$ in the dense ionized gas of high-redshift quasi-stellar objects where $\mathrm{HeH}^{+}$production could be increased by local energetic processes, such as collisional excitations or even X-rays excitations, but it was not conclusive either.

It should be stressed that the non-observation of $\mathrm{HeH}^{+}$is similarly true of the giant planets, a medium in which hydrogen and helium are also abundant with the presence of a strong source of ionization due to the solar wind. $\mathrm{No} \mathrm{HeH}^{+}$has ever been detected even in the ionosphere, auroral ring, and polar haze of Jupiter, whereas $\mathrm{H}_{3}^{+}$is found to be a major component (Drossart et al. 1989; Maillard et al. 1990). Regarding a system like the PN NGC 7027 the non-detection might be explained by its rapid destruction in contact with $\mathrm{H}_{2}$ (Orient 1977). The expanding ionized shell developed around the central white dwarf where $\mathrm{HeH}^{+}$could form, remains surrounded in this young PN by a molecular envelope, which starts at a distance from the very hot star where $\mathrm{H}_{2}$ is no longer dissociated. This structure has been well shown by the observations of NGC 7027 in the $\mathrm{Br} \gamma$ line and the 1-0 S(1) line of $\mathrm{H}_{2}$ by Cox et al (2002).

This non-observation, even in hydrogen rich regions, could imply that $\mathrm{HeH}^{+}$is not a proper target and that alternatives have to be considered, for example small $\mathrm{HeH}_{n}^{+}$clusters. In other words, the question of the search of hydrogen-helium ions, originally focused on $\mathrm{HeH}^{+}$, might be reconsidered as the search for $\mathrm{HeH}_{3}^{+}$the simpler cluster. The question is more current than ever since the synthesis of this complex in a $3.7 \mathrm{~K}$ ion trap by Savić et al (2015).

In this work, we reconsider the question at a level of theory using highly correlated wave functions, which we believe to be necessary. The method employed for the electronic calculations is briefly presented in Sect. 2. The different steps of the approach leading to the determination of the potential energy surface (PES) are shown in Sect. 3, followed by the calculation of the rate constants for the associated radiative association reactions leading to the $\mathrm{HeH}_{3}^{+}$complex (Sect. 4). The possible consequences of this study are discussed in Sect. 5 .

\section{Computational background}

All the electronic calculations were performed using a CASPT2 approach (Andersson et al. 1990, 1992). This two-step procedure starts with a complete active space self-consistent calculation (CAS) in which all possible configurations that satisfy the spin and symmetry constraints are included in the orbitals optimization. This first step is carried out to include the chemically important part of the electron correlation energy. The second step is a second order perturbation treatment (PT2) of the CAS wave function designed to recover the larger part of the dynamic correlation energy missing after the first step of the procedure. The atomic basis set is the ANO-L-VQZP (Widmark et al. 1990; Widmark et al. 1991; Pou-Amérigo et al. 1995; Pierloot et al. 1995), which includes relativistic effects. All calculations were performed by means of the MOLCAS program (Aquilante et al. 2010).

A series of test calculations was first carried out around the equilibrium geometry of $\mathrm{H}_{3}^{+}$to determine the dimension of the active space to be used. All active spaces were generated by distributing the four active electrons, all possible ways, in a set of orbitals of increasing dimension (Table 1).

We found that active space 3 was necessary for a steady description of the ${ }^{1} A_{1}$ ground state. Consequently, active space 3 was employed in subsequent calculations performed within $\mathrm{C}_{2 v}$ symmetry.

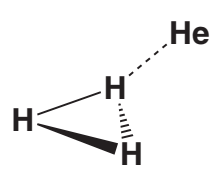

GS<smiles>[CH][CH]</smiles><smiles>[CH]C=C</smiles>

$\mathrm{TS}_{1}$

$\mathrm{TS}_{2}$

Fig. 1. Stationary points on the $\mathrm{He}-\mathrm{H}_{3}^{+}$potential energy surface.

Table 1. Definition of active spaces for the $\left[\mathrm{HeH}_{3}\right]^{+}$system.

\begin{tabular}{lccc}
\hline \hline $\begin{array}{l}\text { Active } \\
\text { space }\end{array}$ & $\begin{array}{c}\mathrm{He} \\
\text { orbitals }\end{array}$ & $\begin{array}{c}\mathrm{H} \\
\text { orbitals }\end{array}$ & Dimension \\
\hline 1 & $1 \mathrm{~s}$ & $1 \mathrm{~s}$ & 4 \\
2 & $1 \mathrm{~s} 2 \mathrm{~s} 2 \mathrm{p}_{\mathrm{x}} 2 \mathrm{p}_{\mathrm{y}} 2 \mathrm{p}_{\mathrm{z}}$ & $1 \mathrm{~s}$ & 8 \\
3 & $1 \mathrm{~s} 2 \mathrm{~s} 2 \mathrm{p}_{\mathrm{x}} 2 \mathrm{p}_{\mathrm{y}} 2 \mathrm{p}_{\mathrm{z}}$ & $1 \mathrm{~s} 2 \mathrm{~s} 2 \mathrm{p}_{\mathrm{x}} 2 \mathrm{p}_{\mathrm{y}} 2 \mathrm{p}_{\mathrm{z}}$ & 20 \\
\hline
\end{tabular}

Table 2. Relative energies $(\mathrm{eV})$ and geometric parameters $(\AA)$ for the $\left[\mathrm{HeH}_{3}\right]^{+}$complex and constitutive fragments.

\begin{tabular}{lccccc}
\hline \hline & $\mathrm{GS}$ & $\mathrm{TS}_{1}$ & $\mathrm{TS}_{2}$ & $\mathrm{HeH}^{+}$ & $\mathrm{H}_{3}^{+}$ \\
\hline$\Delta \mathrm{E}$ & 0.0 & 0.017 & 0.026 & & \\
$d(\mathrm{He}-\mathrm{H})$ & 1.789 & 2.213 & 2.509 & 0.776 & \\
$d(\mathrm{H}-\mathrm{H})_{\mathrm{H}_{3}^{+}}$ & 0.874 & 0.873 & 0.874 & & 0.873 \\
\hline
\end{tabular}

\section{Potential energy surface of $\left[\mathrm{HeH}_{3}\right]^{+}$}

Several studies have already examined the geometry and stability of $\mathrm{HeH}_{3}^{+}$(Funke et al. 1967; Benson \& McLaughlin 1972; Poshusta 1969, 1973). The accurate investigations by Raynor $\&$ Herschbach (1983) yield a $C_{2 v}$ structure with a very low binding energy $(0.04 \mathrm{eV})$ with respect to the $\mathrm{He}+\mathrm{H}_{3}^{+}$fragments. However, not all of the aforementioned studies gave this result; some of these works suggested that the complex would not even be stable. Seeing these contradictions and the spread of the reported results, high level calculations were deemed necessary to obtain a conclusive answer as to the stability and geometry of this complex.

Three geometries were found to be stationary points, two of which belonging to $\mathrm{C}_{2 v}$ and one to $\mathrm{C}_{3 v}$ symmetries (Fig. 1).

There is only one stable structure, GS, one transition state, $\mathrm{TS}_{1}$, implying the in-plane rotation of He from one apex of the triangle to another, and one long distance out-of-plane transition state $\mathrm{TS}_{2}$. The values obtained at the CASPT2/ANO-L-VQZP level are given in Table 2, where $\mathrm{d}(\mathrm{H}-\mathrm{H})_{\mathrm{H}_{3}}^{+}$is the average over the three distances defining the $\mathrm{H}_{3}^{+}$triangle. This result shows that $\mathrm{He}-\mathrm{H}_{3}^{+}$falls in line with all $\mathrm{X}-\mathrm{H}_{3}^{+}(\mathrm{X}=\mathrm{Ne}, \mathrm{Ar}, \mathrm{Kr}$, and $\mathrm{Xe})$ investigated in previous studies (Pauzat \& Ellinger 2007; Pauzat et al. 2009).

Comparison with the values obtained for $\mathrm{HeH}^{+}$and $\mathrm{H}_{3}^{+}$in isolation (Table 2) shows that the $\mathrm{H}_{3}^{+}$cycle is practically the same as in isolation, while the $\mathrm{He}-\mathrm{H}$ bond is considerably longer than for isolated $\mathrm{HeH}^{+}$. This allows us to state that the complex formed corresponds to a very weak interaction between $\mathrm{He}$ and $\mathrm{H}_{3}^{+}$contrary to the strong bond in $\mathrm{HeH}^{+}$. There is no hydrogen transfer from $\mathrm{H}_{3}^{+}$to $\mathrm{He}$, which is confirmed by a topological analysis (Silvi \& Savin 1994) of the electronic structure. As shown in Fig. 2, there is no bonding between the two fragments of the complex whereas a strong interaction (a single electronic 


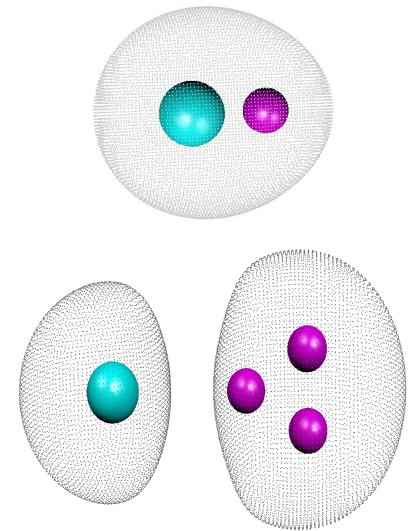

Fig. 2. View of the electron localization function $(E L F=0.85)$ isosurfaces for $\mathrm{HeH}^{+}$and $\mathrm{He}-\mathrm{H}_{3}^{+}$.

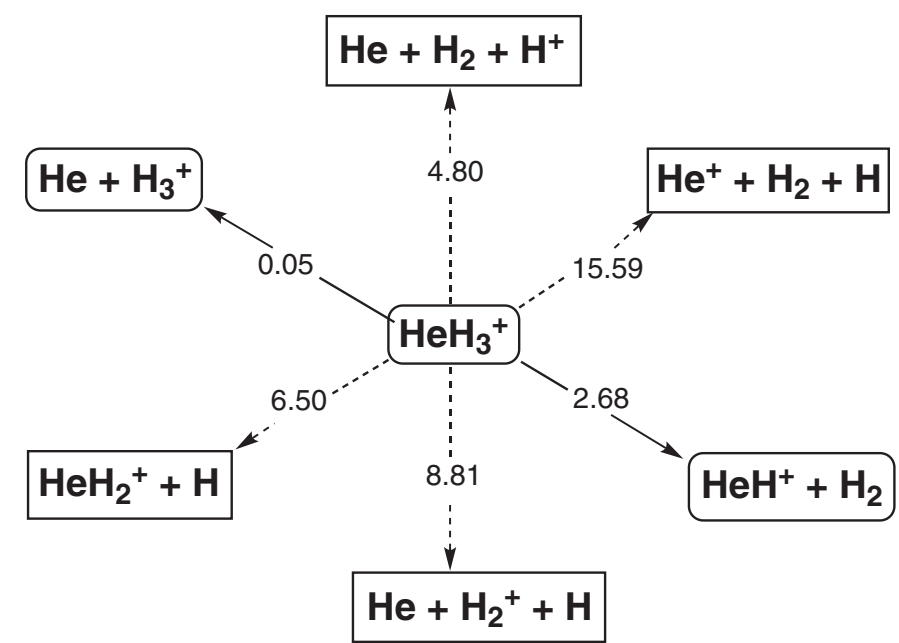

Fig. 3. Energetics $(\mathrm{eV})$ of the fragmentations $\mathrm{He}-\mathrm{H}_{3}^{+}$.

domain) is seen for the diatomic ion. Full details on the method and comparison with other noble gases complexes with $\mathrm{H}_{3}^{+}$can be found in Pauzat \& Ellinger (2007).

Because the geometry of the complex is known, the reaction network representing all the possible constructions and fragmentations of $\mathrm{HeH}_{3}^{+}$was investigated. The results reported in Fig. 3 confirm that the $\mathrm{HeH}_{3}^{+}$complex is the lowest energy point on the PES, where all possible destructions of the complex are endothermic.

At the same time, this schematic representation highlights the pivotal role of $\mathrm{HeH}_{3}^{+}$and points to the lowest energy reaction paths (plain arrows between rounded rectangles). However, more complete calculations are necessary than this only energetic profile to conclude whether $\mathrm{HeH}_{3}^{+}$can be effectively reached or not. This leads us to study two cuts of the PES, corresponding to reaction paths (1) and (2) as follows:

$$
\begin{aligned}
\mathrm{He}+\mathrm{H}_{3}^{+} & \rightarrow \mathrm{HeH}_{3}^{+} \\
\mathrm{HeH}^{+}+\mathrm{H}_{2} & \rightarrow \mathrm{HeH}_{3}^{+} .
\end{aligned}
$$

Reaction (1) amounts to scanning the distance $\mathrm{d}_{1}$ between $\mathrm{He}$ and the closest apex $\mathrm{H}$ and for reaction (2) to scanning the distance $\mathrm{d}_{2}$ between the closest apex $\mathrm{H}$ and the middle of the $\mathrm{H}_{2}$ fragment (assuming $\mathrm{C}_{2 v}$ symmetry; cf. Fig. 4 (top)). The overall result mapped in Fig. 4 (bottom) shows unambiguously that reaction (1) leads to the formation of $\mathrm{He}+\mathrm{H}_{3}^{+}$without any activation barrier. Indeed, it is clearly visible that a start from the entrance
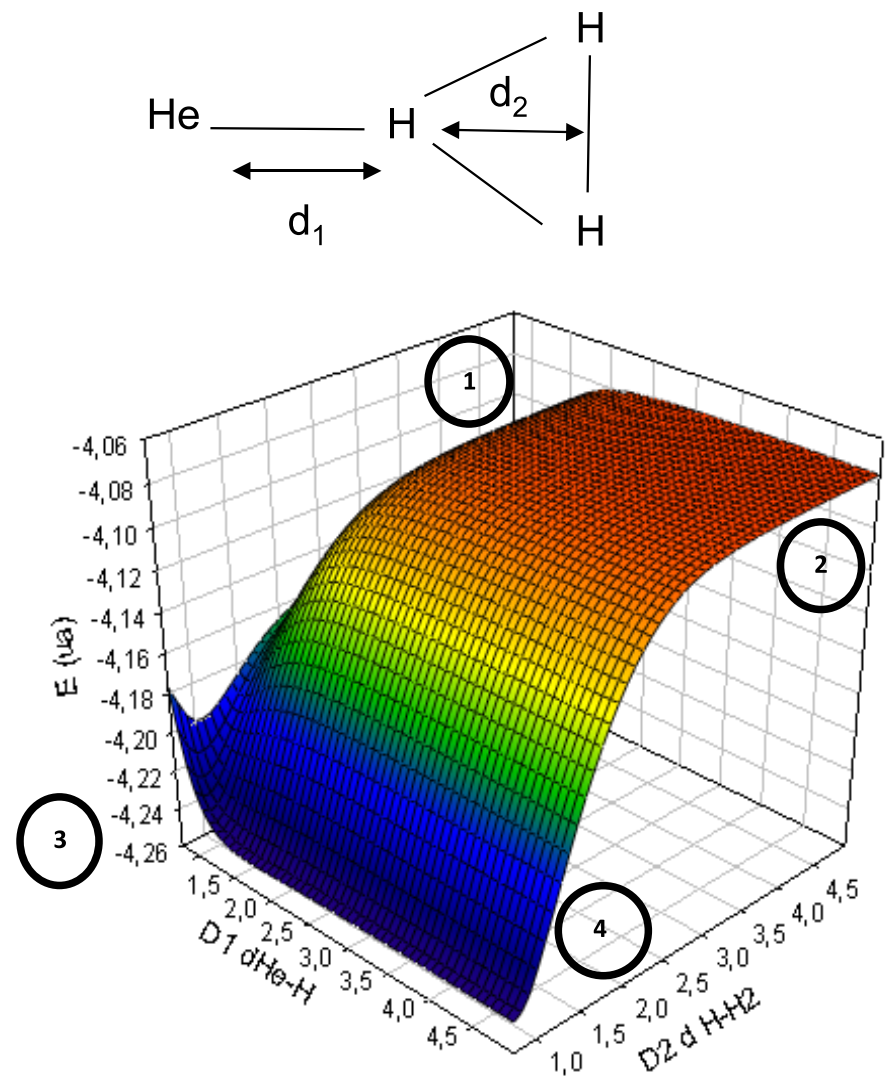

Fig. 4. Top: two dimensions defining the potential energy surface explored. Bottom: CASPT2 potential energy surface; Zone 1: $\mathrm{HeH}^{+}+\mathrm{H}_{2}$; Zone 2: $\mathrm{He}^{+}+\mathrm{H}+\mathrm{H}_{2}$; Zone 3: $\mathrm{HeH}_{3}^{+}$; Zone 4: $\mathrm{He}+\mathrm{H}_{3}^{+}$.

channel considered, namely $\mathrm{HeH}^{+}+\mathrm{H}_{2}$ corresponding to zone 1 leads to a steep descent towards zone 3 . In the same way, starting from zone 4, where the system is under the form $\mathrm{He}+\mathrm{H}_{3}^{+}$, the association also proceeds without activation barrier.

\section{Formation of $\mathrm{HeH}_{3}^{+}$by radiative association}

Since no low-lying excited electronic states are accessible, each mechanism for these radiative associations implies the formation of a complex situated in the vibrational quasi-continuum of the ground electronic state; this complex is then stabilized by radiative decay towards the bound rovibrational levels of the ground state, which releases the excess energy through spontaneous emission. The calculations were carried out here in a reduced dimension quantum approach used previously by Bacchus-Montabonel et al. (2000). In the original approach of atom-atom collision (Zygelman \& Dalgarno 1990; Stancil et al. 1993), the quantum expression of the cross section at a given relative collision energy $E$ of the colliding fragments is given by

$\sigma(E)=\sum_{J} \sigma_{J}(E)$

where

$\sigma_{J}(E)=\sum_{v^{\prime} J^{\prime}} \frac{64}{3} \frac{\pi^{5}}{c^{3}} \frac{g}{2 \mu E} v_{E, v^{\prime} J^{\prime}}^{3} S_{J J^{\prime}} M_{E J, v^{\prime} J^{\prime}}^{2}$,

where $\mu$ is the reduced mass of the system, $g$ is a parameter accounting for the degeneracy of the electronic state (here $g=1$ ), 
Table 3. Radiative association rate coefficients $\left(\mathrm{cm}^{3} \mathrm{~s}^{-1}\right)$ for $\mathrm{He}+\mathrm{H}_{3}^{+}$ $\rightarrow \mathrm{HeH}_{3}^{+}$and $\mathrm{HeH}^{+}+\mathrm{H}_{2} \rightarrow \mathrm{HeH}_{3}^{+}$.

\begin{tabular}{ccc}
\hline \hline$T(\mathrm{~K})$ & $\mathrm{He}+\mathrm{H}_{3}^{+}$ & $\mathrm{HeH}^{+}+\mathrm{H}_{2}$ \\
\hline 10 & $1.51 \times 10^{-23}$ & $3.10 \times 10^{-19}$ \\
20 & $1.74 \times 10^{-23}$ & $6.82 \times 10^{-19}$ \\
30 & $1.68 \times 10^{-23}$ & $1.07 \times 10^{-18}$ \\
40 & $1.53 \times 10^{-23}$ & $1.38 \times 10^{-18}$ \\
50 & $1.37 \times 10^{-23}$ & $1.61 \times 10^{-18}$ \\
60 & $1.21 \times 10^{-23}$ & $1.78 \times 10^{-18}$ \\
70 & $1.08 \times 10^{-23}$ & $1.89 \times 10^{-18}$ \\
80 & $9.64 \times 10^{-24}$ & $1.96 \times 10^{-18}$ \\
90 & $8.66 \times 10^{-24}$ & $2.00 \times 10^{-18}$ \\
100 & $7.82 \times 10^{-24}$ & $2.01 \times 10^{-18}$ \\
200 & $3.59 \times 10^{-24}$ & $1.61 \times 10^{-18}$ \\
300 & $2.14 \times 10^{-24}$ & $1.18 \times 10^{-18}$ \\
500 & $1.07 \times 10^{-24}$ & $7.10 \times 10^{-19}$ \\
1000 & $4.00 \times 10^{-25}$ & $3.06 \times 10^{-19}$ \\
\hline
\end{tabular}

$S_{J J^{\prime}}$ is the Höln-London coefficients characteristic of the $J$ and $J^{\prime}$ pair of rotational levels, and $M_{E J, v^{\prime} J^{\prime}}^{2}$ is the free-bound transition moment matrix element of the dipole moment between the energy normalized function $f_{E J}$ of the continuum and the bound rovibrational function $\phi_{v^{\prime} J^{\prime}}$. The frequency of the photon emitted, $v_{E, v^{\prime} J^{\prime}}$, corresponding to the energy difference between the continuum level of energy $E$ and the rovibrational bound state of energy $E_{v^{\prime} J^{\prime}}$ is given by

$\Delta E=h v_{v^{\prime} J^{\prime}}=E-E_{v^{\prime} J^{\prime}}$.

Finally, the rate constants are calculated as the average over the cross sections weighted with a Maxwellian velocity distribution at temperature T (Stancil et al. 1993; Juřek et al. 1995; Gianturco \& Gori-Giorgi 1997), i.e.

$k(T)=\langle\sigma(E) v\rangle$.

For diatomic systems, it is possible to determine both the rovibrational bound states and the corresponding free-bound dipole transition moments so that a rigorous quantum chemical treatment can be carried out as presented recently for the formation of $\mathrm{CHe}^{++}$by radiative association (Zicler et al. 2016). Following Bates (1987) and Herbst \& Bates (1988) this treatment was adapted in a reduced dimension via a one-dimensional reaction coordinate approximation. In this context, the $\mathrm{HeH}_{3}^{+}$excited complex is formed along the $\mathrm{d}_{1}$ and $\mathrm{d}_{2}$ for reactions (1) and (2), respectively.

The energy curve necessary to the calculation of the rate constant for the $\mathrm{He}-\mathrm{H}_{3}^{+}$association is obtained by the cut of the global PES along $d_{1}$ shown in Fig. 4. The geometry is relaxed for every value of the reaction coordinate to account for the rearrangement of the $\mathrm{H}_{3}^{+}$fragment when approaching the helium atom. In that case the potential energy curve presents a very small well, only $0.051 \mathrm{eV}$ (i.e. $1.17 \mathrm{kcal} \mathrm{mol}^{-1}$ ) deep, with three vibrational levels $(v=0$ to 2$)$. Thus the radiative association rate constants obtained (Table 3 ) remain very weak in the whole temperature domain, reaching $1.7 \times 10^{-23} \mathrm{~cm}^{3} \mathrm{~s}^{-1}$ at most. The stabilization of the $\mathrm{HeH}_{3}^{+}$complex through the rovibrational levels cascade would thus be very unlikely.

In the same way, the cut according to $\mathrm{d}_{2}$ (Fig. 4) with both $\mathrm{HeH}^{+}$and $\mathrm{H}_{2}$ fragments relaxed to account for their rearrangements is used for the calculation of the $\mathrm{HeH}^{+}-\mathrm{H}_{2}$ association. In this reaction, the stabilization of the $\mathrm{HeH}_{3}^{+}$complex would be much more likely. The depth of the potential energy well is $2.62 \mathrm{eV}$ (i.e. $60.3 \mathrm{kcal} \mathrm{mol}^{-1}$ ) and up to 28 vibrational levels $(v=0$ to 27) are found in this case. The radiative association cross sections (Table 3 ) have been summed over all the rovibrational levels up to $J=12$ and the corresponding rate coefficients reach up to $2.0 \times 10^{-18} \mathrm{~cm}^{3} \mathrm{~s}^{-1}$ meaning, obviously, a more efficient process.

\section{Concluding remarks}

As already stated in the introduction, the non-observation of $\mathrm{HeH}^{+}$in various hydrogen-rich media has drawn much attention. Recently, Loreau et al. (2013) revisited this fact by considering an alternative approach through excited states of $\mathrm{HeH}^{+}$and proposed that this ion could be detected in its metastable ${ }^{3} \Sigma^{+}$state. Evaluation of the rate constant of its possible formation by radiative association between metastable helium and a proton gave values in the range of $\sim 10^{-16} \mathrm{~cm}^{3} \mathrm{~s}^{-1}$. This evaluation, however, was penalized by the short live times and low abundances of the excited states. On the basis of these results, these authors estimated the abundance of the $\mathrm{HeH}^{+}$excited triplet and found it to be much lower than that of the ground state.

By contrast, all our developments are concerned with ground electronic states for all reactants and products. Even if the geometry of the fragments is allowed to adjust along the reaction paths, the calculations still suffer the consequences of the reduced dimension model, although they are performed in a quantum framework. This formulation is not able, indeed, to account for all the degrees of freedom that could help in dissipating the energy via the radiative emission. In other words, the association energy cannot spread over all the internal rotational and vibrational modes associated with the remaining $\mathrm{HeH}^{+}$and $\mathrm{H}_{3}^{+}$ bonds, such that the radiative rates in Table 3 should be somehow underestimated. Nevertheless, the present treatment offers a first approach to the vibrational relaxation in the $\mathrm{HeH}_{3}^{+}$formation processes considering the effect of the directly coupled motions on the interaction potential along the reaction coordinate, which is reasonable according previous work (Kunc 1998; BacchusMontabonel et al. 2000; Patuwo et al. 2013). In the end, our calculated rate coefficients that reach up to $2.0 \times 10^{-18} \mathrm{~cm}^{3} \mathrm{~s}^{-1}$ suggest that the formation of $\mathrm{HeH}_{3}^{+}$should not be a priori discarded, meaning that it is worth searching for this molecular ion.

The question raised now is where to look for these ionic species in space since both are known to exist in the laboratory. The detection of $\mathrm{HeH}^{+}$seems to be rather unlikely, taking into consideration its straightforward destruction by $\mathrm{H}_{2}$ (Orient 1977). The planetary nebula in which $\mathrm{HeH}^{+}$has been looked for (NGC 7027) has a rich $\mathrm{H}_{2}$ envelope, which does not make it a good environment for $\mathrm{HeH}^{+}$to be preserved and observed. Thus, maybe PNs that are old and evolved enough to have lost their $\mathrm{H}_{2}$ envelope, such as the Ring Nebula (M57), might be better targets. However, this observation has never been tried owing to the lack of $\mathrm{N}$ appropriate instrument. It would require a wide ( $\gg 1$ arcmin), integral field spectrometer in the $3 \mu \mathrm{m}$ spectral region which, so far, does not exist. However, this last test should be kept in mind before a final conclusion.

As suggested in this paper, another chance to find a molecular ion with $\mathrm{He}$, would be to look for $\mathrm{HeH}_{3}^{+}$. In such a case, the first point is to reference the places where $\mathrm{H}_{3}^{+}$has been observed. Indeed, $\mathrm{H}_{3}^{+}$was first detected in the auroral zones of Jupiter (Drossart et al. 1989) and then, in those of Saturn and Uranus (Maillard \& Miller 2011). This fundamental ion is formed there by the particle flux of the solar wind on $\mathrm{H}_{2}$ reaching the upper atmosphere of the polar regions. It will be destroyed deeper by 
reaction with $\mathrm{H}_{2}$, but its lifetime is long enough to survive in sufficient quantity to be detectable in emission, as the signature of an auroral activity on the giant planets. However, the chance of having the formation of the complex with He seems unlikely, as the helium atoms, submitted to the gravity effect, sink into the lower atmosphere of these planets, making He depleted in the upper atmosphere where $\mathrm{H}_{3}^{+}$forms.

The central molecular zone (CMZ) represents another site to consider. Recent papers by Goto (2015a) and Oka et al. (2016) have pointed out that the central $300 \mathrm{pc}$ of the Galaxy exhibit a diffuse emission of $\mathrm{H}_{3}^{+}$all over the region. Another paper (Goto et al. 2013) is devoted to the $\mathrm{H}_{3}^{+}$spectroscopy of the central few parsecs. On the other hand, a few years before, evidence for a cluster of helium-rich stars (Paumard et al. 2001) and flows of helium coming from the abundant mass loss of these stars (Paumard et al. 2004) have been found in the inner parsecs. Could the conditions for having there an efficient formation of $\mathrm{HeH}_{3}^{+}$be satisfied? Helium is abundant in the inner region, while $\mathrm{H}_{3}^{+}$forms in the surrounding molecular zone; this forms the CMZ, which is submitted to the ionization of the cosmic rays. The detection of $\mathrm{H}_{3}^{+}$in this region is made in absorption, on the line of sight of the bright stars concentrated in the vicinity of the central black hole, in particular the helium-rich stars (Paumard et al. 2001). The $\mathrm{H}_{3}^{+}$spectra presented in Goto et al. (2013) show five or six weak broad line profiles, about $200 \mathrm{~km} \mathrm{~s}^{-1}$ wide, marks of gas flows within the CMZ, with a maximum depth of $10 \%$. The search for potential $\mathrm{HeH}_{3}^{+}$lines should be carried out with the same method. The very likely small production rate of $\mathrm{HeH}_{3}^{+}$leads to foresee lines that are too weak to be easily detectable. In addition, the temperature of this area, close to the central black hole, might be too high for $\mathrm{HeH}_{3}^{+}$to survive durably, when considering the experimental results obtained by Savić et al. (2015).

Few star-forming regions are the other sites where $\mathrm{H}_{3}^{+}$has been detected. The first reported detection goes back to a paper of Geballe \& Oka (1996). A more recent work is from Goto et al. (2015b). The method of detection is exactly the same as that used for the CMZ, which consists of looking for the most intense infrared lines of the ion in absorption towards young massive stars embedded in the molecular clouds. The same considerations applied to the CMZ, casting some doubt on the possibility of detecting a signature of $\mathrm{HeH}_{3}^{+}$on the same line of sight.

Nevertheless, it could be challenging to try to observe this compound with the most sensitive high-resolution infrared spectrometers behind a large telescope. In order to help, we tentatively computed the IR spectrum and the rotational constants (Appendix A) of this intriguing positive ion.

Acknowledgements. This work was supported by CNRS national program PCMI (Physics and Chemistry of the Interstellar Medium) and by COST Actions CM 805, "The Chemical Cosmos" and CM 1401, "Our astrochemical history". We acknowledge computational resources from the CCIN2P3 in Villeurbanne.

\section{References}

Andersson, K., Malmqvist, P.-A., Roos, B. O., Sadlej, A. J., \& Wolinski, K. 1990, J. Phys. Chem., 94, 5483

Andersson, K., Malmqvist, P.-A., \& Roos, B. O. 1992, J. Chem. Phys., 96, 1218 Aquilante, F., De Vico, L., Ferré, N., et al. 2010, J. Comput. Chem., 31, 224 Bacchus-Montabonel, M. C., Talbi, D., \& Persico, M. 2000, J. Phys. B, 33, 955
Barlow, M. J., Swinyard, B. M., \& Owen, P. J. 2013, Science, 342, 1343 Bates, D. R. 1987, ApJ, 312, 363

Benson, M. J., \& McLaughlin, D. R. 1972, J. Chem. Phys., 56, 1322 Black, J. H. 1978, ApJ, 222, 125

Bovino, S., Tacconi, M., Gianturco, F. A., \& Galli, D. 2011, A\&A, 529, A140 Bovino, S., Gianturco, F. A., \& Tacconi, M. 2012, Chem. Phys. Lett., 554, 47 Cox, P., Huggins, P. J., Maillard, J. P., et al. 2002, A\&A, 384, 603 Dabrowski, I., \& Herzberg, G. 1978, Ann. N. Y. Acad. Sci., 38, 14 Dalgarno, A., \& Lepp, S. 1987, in Astrochemistry, eds. M. S. Vardya, \& S. P. Tarafdar (Dordrecht, Holland: Reidel), 109

Drossart, P., Maillard, J. P., Caldwell, J., et al. 1989, Nature, 340, 539

Engel, E. A., Doss, N., Harris, G. J., \& Tennyson, J. 2005, MNRAS, 357, 471

Flower, D. R., \& Roueff, E. 1979, A\&A, 72, 361

Frisch, M., Trucks, G. W., Schlegel, H. B., et al. 2009, Gaussian 09 (Wallingford, CT: Gaussian, Inc.), 227

Funke, I., Preuss, H., \& Diercksen, G. 1967, Mol. Phys., 13, 517

Geballe, T. R., \& Oka, T. 1996, Nature, 384, 334

Gianturco, F., \& Gori-Giorgi, P. 1997, ApJ, 479, 560

Goto, M., Indriolo, N., Geballe, T. R., \& Usuda, T. 2013, J. Phys. Chem. A, 117, 9919

Goto, M., Geballe, T. R., \& Usuda, T. 2015a, ApJ, 806, 57

Goto, M., Geballe, T. R., Usuda, T., et al. 2015b, AIP Conf. Proc., 1642, 377

Gruenwald, R. B., \& Péquignot, D. 1989, Planetary Nebulae, ed. S. Torres-

Peimbert (Dordrecht, Holland: Kluwer), 224

Harris, G. J., Lynas-Gray, A. E., Miller, S., \& Tennyson, J. 2004, ApJ, 617, L143

Herbst, E., \& Bates, D. R. 1988, ApJ, 329, 410

Hirasawa, T. 1969, Prog. Theor. Phys., 42, 523

Hogness, T. R., \& Lunn, E. G. 1925, Phys. Rev., 26, 44

Juřek, M., Spirko, V., \& Kraemer, W. P. 1995, Chem. Phys., 193, 287

Kunc, J. A. 1998, Phys. Rev. E, 58, 4960

Lepp, S., Stancil, P. C., \& Dalgarno, A. 2002, J. Phys. B, 35, R57

Loreau, J., Vranckx, S., Desouter-Lecomte, M., Vaeck, N., \& Dalgarno, A. 2013, J. Chem. Phys., 117, 9486

Liu, X.-W., Barlow, M. J., Tennyson, J., et al. 1997, MNRAS, 290, L71

Maillard, J. P., \& Miller, S. 2011, Molecules in the Atmospheres of Extrasolar Planets, eds. J.-P. Beaulieu, S. Dieters, \& G. Tinetti, ASP Conf. Ser., 450, 19 Maillard, J. P., Drossart, P., Watson, J. K. G., Kim, S. J., \& Caldwell, J. 1990, ApJ, 363, L37

Middlemass, D. 1990, MNRAS, 244, 294

Miller, S., Tennyson, J., Lepp, S., \& Dalgarno, A. 1992, Nature, 355, 420

Moorhead, J. M., Lowe, R. P., Wehlau, W. H., Maillard, J. P., \& Bernath, P. F. 1988, ApJ, 326, 899

Oka, T., Geballe, T. R., Goto, M., et al. 2016, 71st Int. Symp. on Mol. Spec., http://hdl .handle.net/2142/91152

Orient, O. J. 1977, Chem. Phys. Lett., 52, 264

Patuwo, M. Y., Lim, M. B., \& Bettens, R. P. A. 2013, Chem. Phys. Lett., 555, 247

Paumard, T., Maillard, J. P., Morris, M., \& Rigaut, F. 2001, A\&A, 366, 466

Paumard, T., Maillard, J. P., \& Morris, M. 2004, A\&A, 426, 81

Pauzat, F., \& Ellinger, Y. 2007, J. Chem. Phys., 127, 014308

Pauzat, F., Ellinger, Y., Pilmé, J., \& Mousis, O. 2009, J. Chem. Phys., 130, 174313

Pierloot, K., Dumez, B., Widmark, P. O., \& Roos, B. O. 1995, Theor. Chim. Acta, 90, 87

Poshusta, R. D. 1969, J. Chem. Phys., 51, 3343

Poshusta, R. D. 1973, J. Chem. Phys., 59, 2477

Pou-Amérigo, R., Merchan, M., Nebot-Gil, I., Widmark, P.-O., \& Roos, B. O. 1995, Theor. Chim. Acta, 92, 149

Raynor, S., \& Herschbach, D. R. 1983, J. Chem. Phys., 87, 289

Roberge, W., \& Dalgarno, A. 1982, ApJ, 255, 489

Savić, I., Gerlich, D., Asvany, O., Jusko, P., \& Schlemmer, S. 2015, Mol. Phys., 113,2320

Silvi, B., \& Savin, A. 1994, Nature, 371, 683

Stancil, P C., Babb, J. F., \& Dalgarno, A. 1993, ApJ, 414, 672

Widmark, P.-O., Malmqvist, P.-A., \& Roos, B. O. 1990, Theor. Chim. Acta, 77, 291

Widmark, P.-O., Joakim, B., Persson, \& Roos, B. O. 1991, Theor. Chim. Acta, 79, 419

Zicler, E., Bacchus-Montabonnel, M. C., Pauzat, F., Chaquin, P., \& Ellinger, Y. 2016, J. Chem. Phys., 144, 111103

Zinchenko, I., Dubrovich, V., \& Henkel, C. 2011, MNRAS, 415, L78

Zygelman, B., \& Dalgarno, A. 1990, ApJ, 365, 239 


\section{Appendix A}

The harmonic and anharmonic frequencies and associated absolute intensities are reported in Table A.1, as computed using second-order perturbation theory (MP2) according to Frisch et al. (2009). The IR spectrum (Fig. A.1) is dominated by three intense bands. Two of these bands appear at high frequencies, associated with the $\mathrm{A}_{1}$ and $\mathrm{B}_{2}$ modes of the $\mathrm{H}_{3}^{+}$fragment that would be degenerated in absence of the He atom. The third band is at low frequency corresponding to the elongation of the weak bond $\mathrm{He} . \mathrm{H}_{3}^{+}$.

The anharmonic contribution is important as expected for a weakly bound system composed of two fragments of similar masses. The calculated shift amounts to $\sim 9 \%$ in the average for the high frequencies of the $\mathrm{H}_{3}^{+}$fragment. The relative motion between $\mathrm{He}$ and $\mathrm{H}_{3}^{+}$is even more affected by anharmonicity with shifts of more than $50 \%$ of the harmonic value towards lower frequencies. Intensities implying the $v=0$ level follow the same pattern whereas those of overtones and combination bands increase significantly.

In the same way, anharmonicity brings important changes to the rotational constants between those calculated at the equilibrium geometry, i.e.

$$
A_{\mathrm{e}}=1346.8 ; B_{\mathrm{e}}=54.337 ; C_{\mathrm{e}}=52.230(\mathrm{GHz})
$$

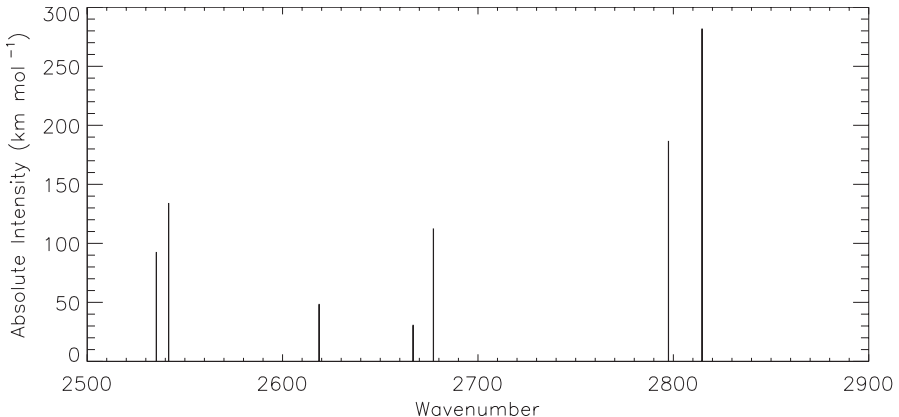

Fig. A.1. Portion of the synthetic spectrum of $\mathrm{HeH}_{3}^{+}$from Table A.1 in which the most intense lines are concentrated, mainly from the fundamental bands associated with the $\mathrm{A}_{1}$ and $\mathrm{B}_{2}$ modes of $\mathrm{H}_{3}^{+}$. The spectral region between 3.45 and $4.00 \mu \mathrm{m}$ should be a top priority for a detection of this ion.

and those obtained when taking into account the vibrational contribution and the centrifugation distortion

$$
A_{0}=1363.0 ; B_{0}=46.582 ; C_{0}=44.196(\mathrm{GHz}) \text {. }
$$

The large corrections on the spectroscopic signatures are an illustration of a very complicated and flat potential surface. It points clearly to the necessity for the evaluation of a proper multi-dimensional potential surface and a full treatment of rovibrational interactions.

Table A.1. Anharmonic infrared spectroscopy of He... $\mathrm{H}_{3}^{+}\left(\mathrm{C}_{2 v}\right.$ symmetry): energies (frequencies $\left(\mathrm{cm}^{-1}\right)$ and absolute intensities $\left(\mathrm{km} \mathrm{mol}^{-1}\right)$ calculated at the MP2 level with aug-cc-pVQZ basis set.

\begin{tabular}{ccccc}
\hline \hline $\begin{array}{c}\text { Fundamental bands } \\
\text { Mode(Quanta) }\end{array}$ & $E($ harm $)$ & $E($ anharm $)$ & $I$ (harm) & $I$ (anharm) \\
\hline $1 \mathrm{~A}_{1}(1)$ & 3488.7 & 3226.6 & 0.6 & 0.2 \\
$2 \mathrm{~A}_{1}(1)$ & 2814.7 & 2535.4 & 281.7 & 92.3 \\
$1 \mathrm{~B}_{2}(1)$ & 2797.5 & 2541.7 & 186.4 & 133.8 \\
$1 \mathrm{~B}_{1}(1)$ & 287.5 & 103.5 & 6.0 & 4.6 \\
$3 \mathrm{~A}_{1}(1)$ & 249.2 & 103.7 & 245.3 & 124.4 \\
$2 \mathrm{~B}_{2}(1)$ & 200.9 & 95.6 & 12.8 & 10.1 \\
\hline Overtone bands* & & & & \\
Mode(Quanta) & $E($ harm $)$ & $E($ anharm $)$ & & $I($ anharm $)$ \\
\hline $2 \mathrm{~A}_{1}(2)$ & 5629.5 & 4915.4 & & 27.4 \\
$1 \mathrm{~B}_{2}(2)$ & 5595.1 & 4912.2 & & 33.2 \\
$3 \mathrm{~A}_{1}(2)$ & 498.4 & 125.9 & & 13.0 \\
$2 \mathrm{~B}_{2}(2)$ & 401.9 & 115.3 & & 2.9 \\
\hline Combination bands $^{*}$ & & & & \\
Mode(Quanta) & $E($ harm $)$ & $E($ anharm $)$ & & 112.2 \\
\hline $2 \mathrm{~B}_{2}(1) \mid 1 \mathrm{~B}_{2}(1)$ & 2998.5 & 2677.2 & & 49.3 \\
$2 \mathrm{~A}_{1}(1) \mid 1 \mathrm{~B}_{2}(1)$ & 5612.3 & 4993.9 & & 48.3 \\
$2 \mathrm{~A}_{1}(1) \mid 2 \mathrm{~B}_{2}(1)$ & 3015.7 & 2618.7 & & 1.4 \\
$2 \mathrm{~A}_{1}(1) \mid 1 \mathrm{~A}_{1}(1)$ & 6303.4 & 5582.3 & & 30.6 \\
$1 \mathrm{~B}_{1}(1) \mid 2 \mathrm{~A}_{1}(1)$ & 3102.3 & 2666.8 & & \\
\hline
\end{tabular}

Notes. * Only bands with $I \geq 1$ are reported. 